\title{
Influência da temperatura de secagem do resíduo de Pacú (Piaractus mesopotamicus) para obtenção de óleo
}

Os resíduos gerados na pesca apresentam altas concentrações de óleo, que pode ser reaproveitado para produção de biodiesel, desenvolvimento de novos produtos voltados para alimentação humana ou fármacos. A temperatura de secagem influencia significativamente na degradação do óleo obtido, interferindo na qualidade do mesmo. Diante disso, o presente estudo objetivou avaliar a cinética de secagem de resíduo de pacu, e a caracterização do óleo obtido em relação ao índice de acidez. As amostras do resíduo de pacu trituradas foram submetidas a secagem em estufa com circulação de ar forçada nas temperaturas de 50,60 e 70 으, até que atingissem o peso constante das amostras (umidade de equilíbrio). Posteriormente, com as amostras secas, realizou-se a extração do óleo pelo método de soxhlet, utilizando éter de petróleo como solvente e o óleo obtido foi analisado em termos de índice de acidez em ácido oleico (\%). Três modelos matemáticos foram utilizados para o ajuste dos dados experimentais da secagem do resíduo, onde o modelo de Page foi o que apresentou o melhor ajuste às curvas de secagem. Na análise do índice de acidez, verificou-se que o aumento da temperatura de secagem do resíduo ocasionou em menores índices de acidez ou seja, menor degradação do óleo, que foi na temperatura de 70 드, com um índice de acidez de $18,27 \%$. Entretanto, de acordo com a legislação vigente, esse valor ainda se encontra elevado, sendo necessários novos estudos que busquem condições adequadas para secagem e extração do óleo, visando um índice de acidez dentro dos padrões estabelecidos.

Palavras-chave: Cinética; Modelagem Matemática; Índice de Acidez; Soxhlet.

\section{Influence of the drying temperature of Pacú (Piaractus mesopotamicus) residues for oil obtaining}

\begin{abstract}
The waste generated in the fish activity, have high concentrations of oil, which can be reused for biodiesel production or the development of new products for food or drugs. Drying temperature influences greatly the degradation of the oil obtained interfering with the quality of the same. Thus, the present study aimed to evaluate the pacu residue drying kinetics as well as the characterization of the oil obtained in relation to the acidity index. The residue of pacu triturated were submitted to drying in an oven with forced air circulation at temperatures of 50,60 and $70^{\circ} \mathrm{C}$ until they reached a constant weight of the samples (equilibrium moisture content). Subsequently, with the dried samples was carried out extracting oil by soxhlet method, using petroleum ether as solvent and the oil obtained was analyzed in terms of acidity index of oleic acid (\%).Three mathematical models were used to fit the experimental data of drying the waste, where the Page model presented the best fit to the drying curves. In the analysis of acid value, it was found that increasing the drying temperature of the residue resulted in lowe levels of acidity, presenting a smaller degradation of oil, which was at a temperature of $70{ }^{\circ} \mathrm{C}$ with an acid of $18.27 \%$. However, in accordance with current legislation, this value even is high, requiring new studies that aim conditions for drying, as well as different techniques for oil extraction, aiming for an acid value within the established standards.
\end{abstract}

Keywords: Kinetics; Mathematical Modeling; Acid Index; Soxhlet.

Topic: Química Agrícola e Ambiental

Reviewed anonymously in the process of blind peer

\section{César Vinicius Toniciolli Rigueto}

Universidade do Estado de Mato Grosso, Brasil

http://lattes.cnpq.br/5053497434546209

cesartoniciolli@gmail.com

Raquel Aparecida Loss

Universidade do Estado de Mato Grosso, Brasil

http://lattes.cnpq.br/3925129970802016

raquellos@unemat.br

Thainá Stéphanie Martins de Freitas

Universidade do Estado de Mato Grosso, Brasil

http://lattes.cnpq.br/4317716336173183

thainastephanie@hotmail.com
Received: 18/04/2016

Approved: 20/05/2016

Nívia Carolina de Oliveira Bevilaqua

Universidade do Estado de Mato Grosso, Brasi

http://lattes.cnpq.br/5527209552008862

niviabevilaqua@hotmail.com

Fabrício Barros Brum

Universidade do Estado de Mato Grosso, Brasil

http://lattes.cnpq.br/4557152570788817

fabricio b brum@yahoo.com.br
Referencing this:

RIGUETO, C. V. T.; LOSS, R. A.; FREITAS, T. S. M.; BEVILAQUA, N. C. O.; BRUM, F. B.. Influência da temperatura de secagem do resíduo de Pacú (Piaractus mesopotamicus) para obtenção de óleo. Revista Ibero-Americana de Ciências Ambientais, v.8, n.1, p.218-225, 2017. DOI: http://doi.org/10.6008/SPC2179-6858.2017.001.0019 


\section{INTRODUÇÃO}

De acordo com recentes pesquisas realizadas pelo IBGE, o estado de Mato Grosso tem grande destaque na atividade pesqueira do Brasil, sendo o maior produtor de peixes do país, representando $19 \%$ da produção nacional (MUÑOZ et al., 2016). Porém, esta atividade resulta em grandes quantidades de resíduos que são descartados no meio ambiente. Estes resíduos podem apresentar valor agregado, uma vez que são ricos em óleo que pode ser utilizado na alimentação humana ou produção de biodiesel, também de forte atuação na região.

O pacu (Piaractus mesopotamicus) é o segundo peixe nativo mais criado em pisciculturas no país, sendo consumido em maior parte nas regiões Norte e Centro-oeste (GARCIA et al., 2009). Além disso, é um dos peixes que mais se sobressai nos tanques de pisciculturas por proporcionar características desejáveis aos produtores, tais como hábito alimentar onívoro, e carne de boa qualidade (SILVA et al., 2011).

Os resíduos gerados na atividade pesqueira no Brasil em alguns casos podem representar mais de $50 \%$ do peso inicial dos pescados, que são constituídos basicamente pelas vísceras, coluna vertebral, escamas, cauda, barbatana e resquícios de carne (FELTES et al., 2010; COSTA, 2012). Esses resíduos, que são classificados como sólidos orgânicos, quando descartados no meio ambiente, podem levar a uma contaminação direta, devido à fermentação dos ácidos orgânicos, formando o "chorume", além da propagação de vetores e do mau cheiro (RODRIGUES, 2013).

Os ácidos graxos poliinsaturados eicosapentaenóico (EPA) e docosahexaenóico (DHA) são comumente encontrados em pescados e algumas espécies marinhas, apresentando alguns benefícios à saúde, em que o EPA se relaciona com proteção cardiovascular e o DHA no desenvolvimento do cérebro e estrutura ocular do feto durante a gestação (MARTINS et al., 2015). É possível obter estes ácidos graxos a partir do óleo de peixe, sendo que as concentrações mais elevadas de EPA e DHA são encontradas nas vísceras, tornando os resíduos de pescado uma excelente matéria-prima para a extração de óleo (DAUKŠAS et al., 2005).

A análise do índice de acidez apresenta dados a respeito da conservação do óleo, representado como número de miligramas de hidróxido de potássio necessário para que ocorra a neutralização dos ácidos livres presentes em uma grama de amostra. Assim, o índice de acidez revela se está ocorrendo à degradação da cadeia lipídica do óleo, fazendo com que ocorra a liberação de ácidos graxos livres, fornecendo assim, um elevado índice de acidez (SANTOS et al., 2013).

Aguiar (2011) avaliou a extração de óleo de resíduo de filetagem de pescada olhuda (Cynoscion striatus) e explica que o pescado é extremamente perecível devido às características intrínsecas de sua carne, e ainda segundo o mesmo autor, durante o processo de secagem da matéria-prima pode ocorrer à oxidação dos lipídios devido à ação de lipases, existentes no trato gastrointestinal dos peixes. Desta forma, é fundamental estudar condições adequadas de secagem do resíduo de pescado, de maneira a empregar temperaturas que sejam altas o suficiente para inativar a ação enzimática e microbiana, sem a degradação dos lipídios presentes na amostra. 
As informações contidas nas curvas de secagem são de fundamental importância, uma vez que a partir as mesmas é possível estimar o tempo de secagem de uma determinada matéria prima ou produto, além da possiblidade de estimar o gasto energético do processo (REIS et al., 2015). A aplicação de modelos matemáticos às curvas de secagem é uma ferramenta útil, uma vez que a modelagem matemática é uma descrição de um sistema real usando equações matemáticas, que podem ser consideradas simplificações do sistema, baseado em suas características mais significativas (PÉREZ-RODRÍGUEZ; VALERO, 2013).

Diante do exposto, o presente estudo objetivou a avaliação da cinética de secagem dos resíduos de pacu (Piaractus mesopotamicus), bem como a caracterização do óleo obtido por meio do índice de acidez, promovendo o reaproveitamento e produtividade dos recursos naturais, pesqueiros e aquícolas, proporcionando renda extra aos pescadores e agregando valor à sua produção, além de contribuir com o desenvolvimento técnico e cientifico e viabilizar a redução do impacto ambiental.

\section{METODOLOGIA}

\section{Matéria Prima}

Os peixes foram adquiridos de comerciantes locais de Barra do Bugres-MT, permanecendo congelados até ser realizado o processamento. No preparo da matéria-prima, higienizaram-se os peixes com lavagem em água corrente, após, foram filetados para obtenção dos resíduos, constituídos por cabeça, nadadeiras, escamas, cauda, couro e espinhaço (Figura 1). Os resíduos então foram triturados em liquidificador industrial e congelados para posterior secagem.

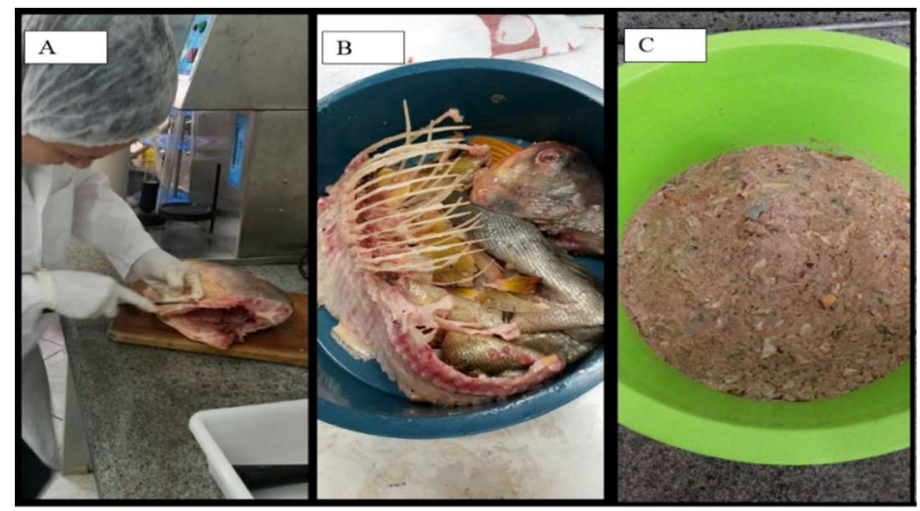

Figura 1: Obtenção dos resíduos de pacu.

A- Evisceração do peixe; B- resíduos dos peixes; C- resíduos processados.

\section{Cinética de Secagem}

A influência da temperatura na qualidade final do óleo foi avaliada submetendo os resíduos de pacu à secagem em estufa com circulação de ar forçada, nas temperaturas de 50, 60 e 70ํㄷ, de acordo com o estudo de Aguiar (2011), que utilizou as mesmas temperaturas para secar resíduo de pescada olhuda. Na secagem da matéria-prima, as pesagens foram realizadas periodicamente, até atingir o peso constante das amostras, e a razão de umidade (RU) foi estabelecida conforma a Equação 1. 


$$
R U=\frac{\left(U-U_{e}\right)}{\left(U_{i}-U_{e}\right)}
$$

Onde:

$\mathbf{U}=$ teor de água do produto, decimal b.s;

Ui= teor de água inicial do produto, decimal b.s; Ue= teor de água de equilíbrio do produto, decimal b.s.

Para a representação das curvas de secagem, utilizou-se três modelos matemáticos empíricos, conforme a Tabela 1. A escolha do melhor modelo que se ajustou aos dados experimentais foi feita em função da correlação e do erro padrão médio.

Tabela1: Modelos matemáticos para a representação da cinética de secagem.

\begin{tabular}{cc}
\hline Modelo & Equação \\
\hline Page & $R U=\exp \left(-k * t^{n}\right)$ \\
Henderson e Pabis & $R U=\mathrm{a} \exp (-k * t)$ \\
Midilli e Kucuk & $R U=\exp \left(-k * t^{n}\right) b * t$ \\
\hline
\end{tabular}

$\mathrm{RU}=$ razão do teor de água do produto, adimensional; $\mathrm{t}=$ tempo de secagem, em $\mathrm{h} ; \mathrm{k}=$ coeficiente de secagem, em $\mathrm{s}^{1} ; \mathrm{a}, \mathrm{b}, \mathrm{n}$ e $\mathrm{k}=$ constante do modelo, adimensional.

\section{Extração do Óleo}

A extração do óleo dos resíduos de pacu foi realizada conforme a técnica de soxhlet descrita pelo Instituto Adolf Lutz (IAL, 2008) com algumas adaptações, utilizando éter de petróleo como solvente. Os balões e cartuchos empregados na extração foram secos em estufas por $24 \mathrm{~h}$, resfriados em dessecador e pesados antes do início da extração. Para cada extração, $5 \mathrm{~g}$ de amostra seca e triturada foi envolvida em um cartucho de papel filtro, o qual foi inserido no extrator. Foram utilizados aproximadamente $150 \mathrm{~mL}$ de solvente, o qual foi acondicionado no balão e aquecido através de uma chapa de aquecimento. $O$ tempo de extração foi de $8 \mathrm{~h}$, e ao final do processo o excesso de solvente foi evaporado em estufa a $70^{\circ} \mathrm{C}$ até peso constante para posterior caracterização.

\section{Caracterização do Óleo de Resíduo de Peixe}

A análise do índice de acidez para cada temperatura de secagem (50, 60 e 70 ㅇ) foi o critério utilizado para caracterizar a qualidade final do óleo. Foram pesados aproximadamente $2 \mathrm{~g}$ de amostra de resíduo de peixe e diluiu-se em $25 \mathrm{~mL}$ de uma solução éter: álcool (2:1). Em seguida foram adicionadas 3 gotas da solução do indicador fenolftaleína e tituladas com solução de hidróxido de sódio 0,01 mol/L (Instituto Adolf Lutz, 2008). As análises das amostras foram realizadas em triplicata e a determinação do índice de acidez foi medida em porcentagem de ácido oléico. Os resultados da acidez foram tratados estatisticamente utilizando o software Statistica, versão 7.0, onde aplicou-se o teste de Tukey $(p<0,05)$ para comparação entre as médias.

\section{RESULTADOS E DISCUSSÃO}

\section{Cinética de Secagem}

No processo de secagem, o tempo que o alimento leva para atingir sua estabilidade máxima depende de algumas variáveis, tais como temperatura, velocidade do ar e dimensões do produto, influindo também 
nas características sensoriais e nutricionais (MARCINKOWSKI, 2006). Assim, para avaliar a influência da temperatura de secagem na qualidade final do óleo extraído os resíduos foram submetidos a secagem em circulação de ar forçado nas temperaturas de 50, 60 e 70 ํ. . As curvas de secagem podem ser observadas na Figura 2.

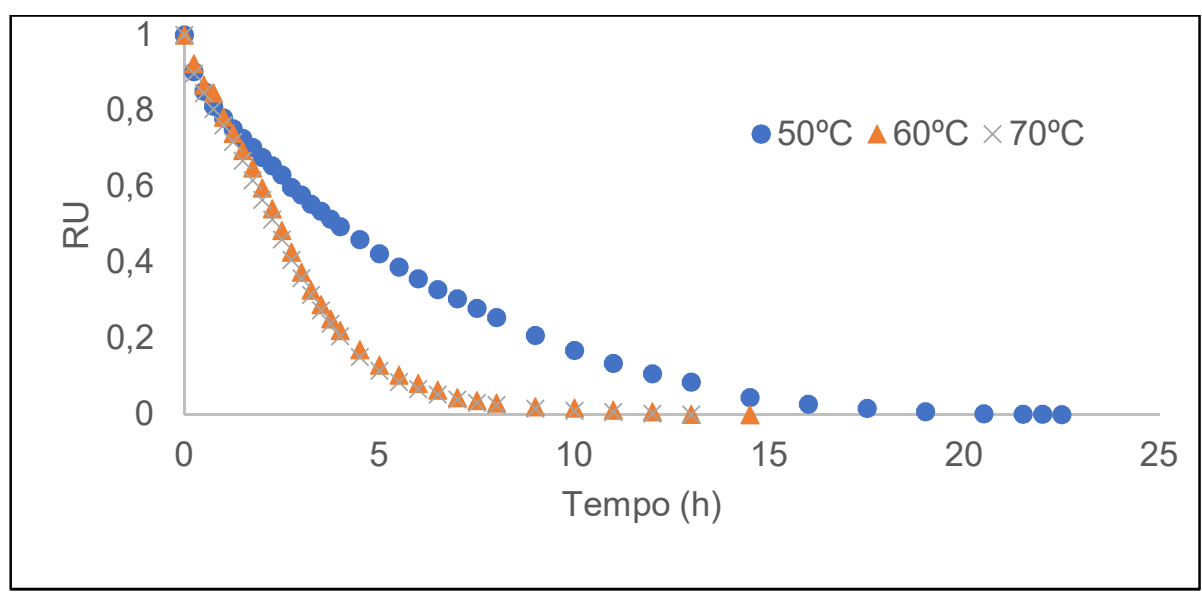

Figura 2: Curvas de secagem do resíduo de pacu a 50, 60 e 70 ㄷ.

Na Figura 2 observa-se o comportamento das curvas de secagem do resíduo de pacu nas três diferentes temperaturas. Verifica-se que em 50 C foram necessárias 20 horas para atingir o peso constante das amostras, já para as temperaturas de 60 e $70 \stackrel{\circ}{ } \mathrm{C}$, nota-se que ambas apresentaram um perfil cinético similar, onde foram necessárias 10 horas, ou seja, metade do tempo das amostras secas em 50 ㄷ․ Entretanto, as massas de água evaporadas não apresentaram diferença significativa, onde foram de 10,62, 10,72 e 10,82 g para 50,60 e $70 \circ \mathrm{C}$, respectivamente.

Utilizando os modelos matemáticos de Henderson e Pabis, Midilli e Kucuk e Page, ajustou-se essas curvas de secagem nas temperaturas de 50,60 e 70 으, os parâmetros ajustados estão apresentados na Tabela 2.

Tabela 2: Ajuste dos parâmetros dos modelos matemáticos de cinética de secagem do resíduo de pacu.

\begin{tabular}{|c|c|c|c|c|}
\hline Modelo & Parâmetros & 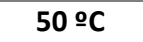 & $60 \circ C$ & 70 ㄷ \\
\hline \multirow{4}{*}{ Henderson e Pabis } & A & 0,940902 & 1,131922 & 1,077386 \\
\hline & k & 0,163011 & 0,385779 & 0,390287 \\
\hline & $r$ & 0,998509 & 0,992183 & 0,993281 \\
\hline & Erro & 4,753198 & 4,350979 & 5,223994 \\
\hline \multirow{6}{*}{ Midilli e Kucuk } & $A$ & 0,884756 & 0,953898 & 0,953552 \\
\hline & $b$ & 0,000000 & 0,000892 & 0,000371 \\
\hline & $\mathrm{k}$ & 0,119086 & 0,182075 & 0,207169 \\
\hline & $\mathrm{n}$ & 1,143876 & 1,477993 & 1,424700 \\
\hline & $r$ & 0,996808 & 0,998351 & 0,998401 \\
\hline & Erro & 0,452796 & 0,312203 & 0,320372 \\
\hline \multirow{4}{*}{ Page } & K & 0,191931 & 0,220761 & 0,231557 \\
\hline & $\mathrm{n}$ & 0,956439 & 1,357858 & 1,363500 \\
\hline & $r$ & 0,997515 & 0,998770 & 0,998481 \\
\hline & Erro & 0,739824 & 0,367114 & 0,381202 \\
\hline
\end{tabular}

Observa-se nos parâmetros da Tabela 2, que dos três modelos aplicados às curvas de secagem nas três temperaturas em estudo, se ajustaram bem aos dados experimentais apresentando correlações acima de 0,99, porém, os modelos de Midilli e Kucuk e de Page apresentaram um menor erro em relação aos 
dados experimentais, logo podemos concluir que estes modelos foram os que melhores se ajustaram. As

Figuras 3a, 3b e 3c, apresentam os ajustes dos modelos aos dados experimentais para as três temperaturas em estudo.
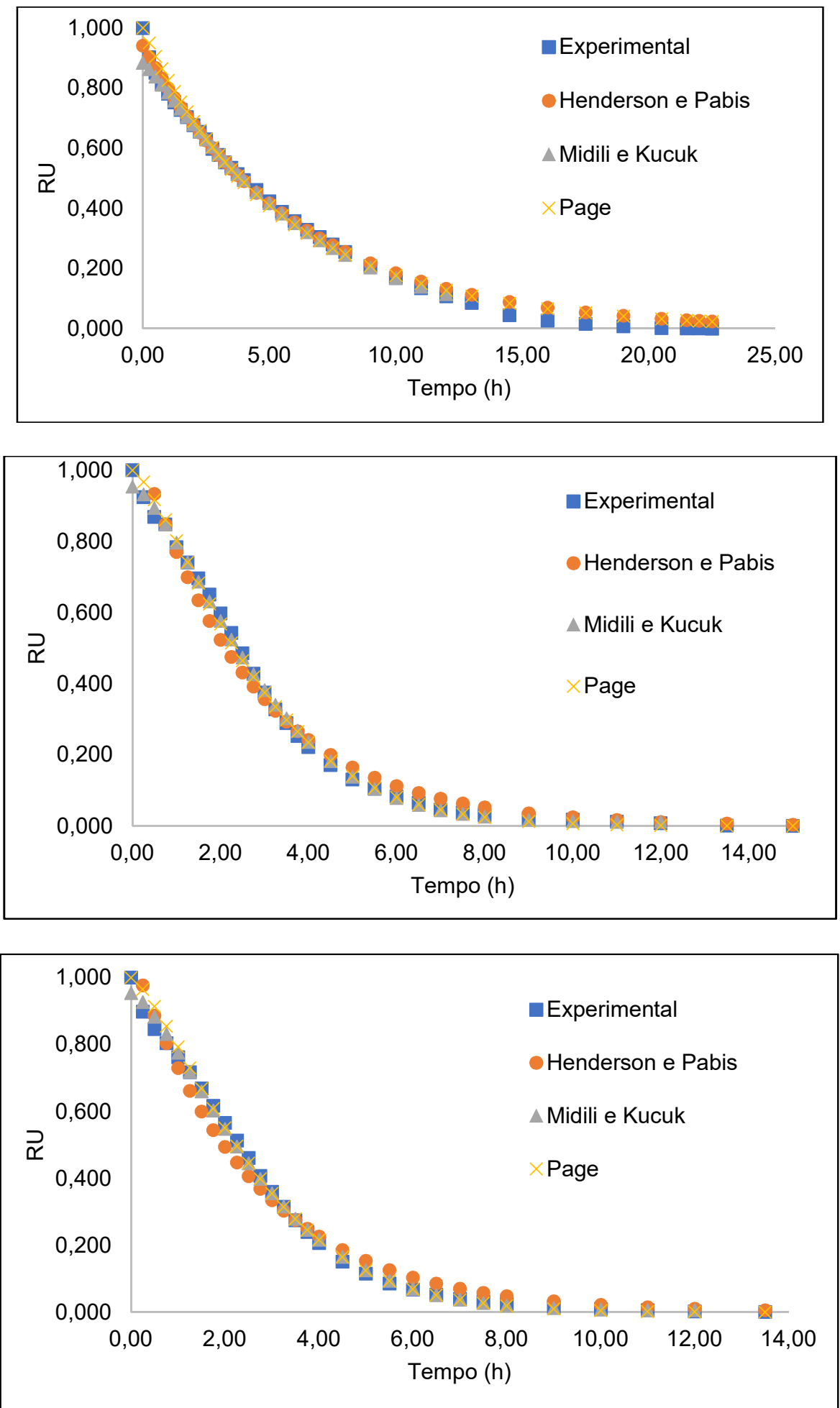

Figura 3: Ajuste dos modelos matemáticos a cinética de secagem do resíduo de pacu nas temperaturas de (a) 50 으 (b) 60 ㅇ C e (c) $70 \circ \mathrm{C}$.

Em outros estudos envolvendo secagem de matérias-primas de origem animal, como os de Djendoubia et al. (2009) com sardinha (Sardina pilchardus), Sobukola e Olatunde (2011) com bagre africano (Clarias gariepinus) e Pezantes (2006) com filés de Bonito (Sarda sarda) salgados e defumados também 
observou-se que o modelo de Page foi o que melhor se ajustou aos dados experimentais. Já Martins et al. (2015) obtiveram como o melhor ajuste para cinética de secagem de pirarucu salgado o modelo de Midilli. Para Deamici et al. (2011) na secagem de silagem de pescado, o modelo de Henderson e Pabis foi o que melhor adequou-se aos dados experimentais.

\section{Caracterização do Óleo de Resíduo de Peixe}

O índice de acidez é realizado no intuito de revelar a condição de conservação dos óleos, sendo que o elevado índice de acidez indica que há quebras na cadeia lipídica do óleo, devido isso ocorre a liberação de seus constituintes como, ácidos graxos (TOFANINI, 2004). A Figura 4 apresenta a acidez em ácido oléico do óleo de peixe extraído via soxhlet do resíduo de pacu submetido a diferentes temperaturas de secagem.

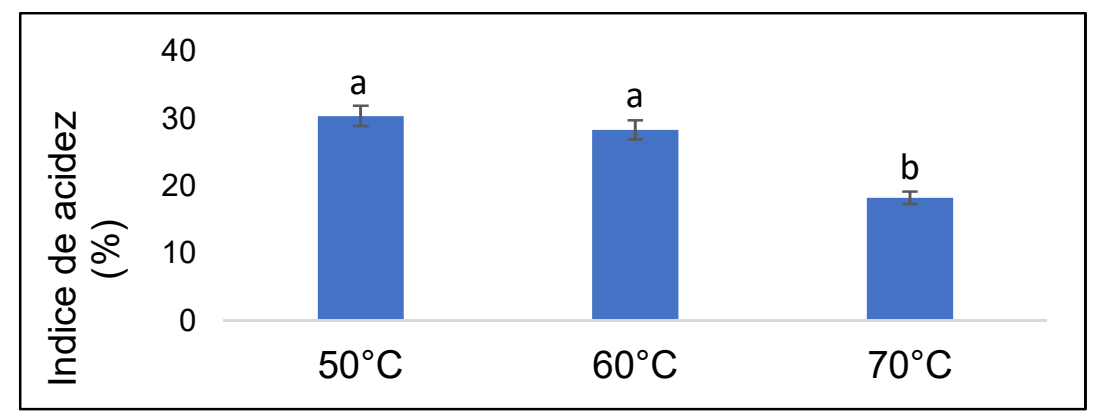

Figura 4: Acidez em ácido oleico do óleo de resíduo de peixe obtido pela extração em soxhlet.

* Letras iguais na mesma coluna não diferem significativamente pelo teste de Tukey $(p<0,05)$.

De acordo com o Regulamento da Inspeção Industrial e Sanitária de Produtos de Origem Animal (RIISPOA) disponível em Brasil (1952) os óleos de pescado devem apresentar acidez em ácido oléico de no máximo 3\%, mostrando assim que os óleos obtidos via extração soxhlet não se encontram de acordo com o índice de acidez determinado pela RIISPOA, uma vez que os valores obtidos são superiores aos 3\%. Além disso, o óleo obtido também não atende as especificações para ser usado como matéria-prima para a produção de biodiesel, uma vez que um óleo com acidez muito elevada impedirá a reação para produção do biodiesel ou o consumo demasiado do catalisador e da mesma forma, um biodiesel com acidez final alta tende a provocar a corrosão do motor ou deterioração do combustível (SILVA et al., 2010).

É importante salientar que apesar do óleo de peixe não ter apresentado o índice de acidez desejado, os resultados obtidos são de extrema importância, uma vez que foi possível observar que a temperatura interfere na qualidade final do óleo, mesmo empregando condições de secagem reportadas na literatura. Outro aspecto que deve ser salientado é o fato que a grande maioria das pesquisas que foram encontradas até o momento na literatura cientifica avaliavam a secagem em função do teor e EPA e DHA e não necessariamente em relação a qualidade final do óleo, o que mostra claramente a contribuição do presente trabalho para a comunidade científica.

\section{CONCLUSÃO}

Avaliando a cinética de secagem nas diferentes temperaturas estudadas é possível concluir que a temperatura de secagem influencia a qualidade final do óleo extraído, onde a amostra seca a $70^{\circ} \mathrm{C}$ forneceu 
um melhor resultado, em termos de índice de acidez. Além disso, na modelagem das curvas de secagem, o modelo de Page foi o que melhor se ajustou aos dados experimentais.

\section{REFERÊNCIAS}

AGUIAR, A. C.. Extração de lipídios de resíduos de filetagem de pescada-olhuda (Cynoscion Striatus) utilizando tecnologia supercrítica. Dissertação (Mestrado em Engenharia de Alimentos) - Universidade Federal de Santa Catarina, Florianópolis, 2011.

BRASIL. Ministério da Agricultura, Pecuária e Abastecimento. Decreto no 30.691, de 29 de março de 1952. Aprova o novo Regulamento da Inspeção Industrial e Sanitária de Produtos de Origem Animal. Brasília: DOU, 1952.

COSTA, S. R.; SOUZA, P. A. R.. O impacto dos resíduos de pescado: o caso da "Feira do Bagaço" no município de Parintins no Amazonas. DELOS Revista Desarrollo Local Sostenible, v.5, n.14, p.1-11, 2012.

DAUKŠAS, E.; FALCH, E.; ŠLIŽYTĖ, R.; RUSTAD, T.. Composition of fatty acids and lipid classes in bulk products generated during enzymic hydrolysis of cod (Gadus morhua) by-products. Process Biochemistry, v.40, n.8, p.2659-2670, 2005.

DEAMICI, K. M.; KRÜMMEL, A.; PEÇANHA, A. D.; DE OLIVEIRA, E. G.. Modelagem semi-empírica da secagem de silagem de pescado. Anais do Salão Internacional de Ensino, Pesquisa e Extensão, v.3, n.2, 2011.

DJENDOUBIA, N.; BOUDHRIOUA, N.; BONAZZIB, C.; KECHAOU, N.. Drying of sardine muscles: Experimental and mathematical investigations. Food and Bioproducts Processing, v.87, n.2, p.115-123, 2009.

FELTES, M. M. C.; CORREIA, J. F. G.; BEIRÃO, L. H.; BLOCK, J. M.; NINOW, J. L.; SPILLER, V. R.. Alternativas para a agregação de valor aos resíduos da industrialização de peixe. Revista Brasileira de Engenharia Agrícola e Ambiental, v.14, n.6, p.669-677, 2010.

GARCIA, F.; MORAES, F. R.; MARTINS, M. L.. Challenge of pacu (Piaractus mesopotamicus) fed diets suplementeal with vitamins $C$ and $E$ by aeromonas hydrophila under diferente temperature. Arquivos brasileiros de Medicina Veterinária e Zootecnia, Belo Horizonte, v.61, n.2, p.378-385, 2009.

IAL. Métodos Físicos Químicos para Análise de Alimentos, 4 ed. Brasília: Instituto Adolfo Lutz, 2008.

MARCINKOWSKI, E. A.. Estudo da cinética de secagem, curvas de sorção e predição de propriedades termodinâmicas da proteína texturizada de soja. Dissertação (Mestrado em Engenharia Química) Universidade Federal do Rio Grande do Sul, Porto Alegre, 2006.
MARTINS, M. G.; MARTINS, D. E. G.; PENA, R. S.. Drying kinetics and hygroscopic behavior of pirarucu (Arapaima gigas) fillet with different salt contents. LWT - Food Science and Technology, v.62, n.1, p.144-151, 2015.

MUÑOZ, A. E. P.; RODRIGUES, A. P. O.; PEDROZA FILHO, M. $X$.. Perspectivas da agropecuária no Brasil e no Mato Grosso. Brasília: Embrapa Pesca e Aquicultura, 2016.

PÉREZ-RODRIGUES, F.; VALERO, A.. Predictive Microbiology in Foods. New York: Springer, 2013.

PEZANTES, D. V.. Estudo das operações combinadas da desidratação osmótica a vácuo, defumação líquida e secagem em filés de bonito (Sarda sarda). Dissertação (Doutorado em Engenharia de Alimentos) - Universidade Estadual de Campinas, Campinas, 2006.

REIS, D. R.; SANTOS, P.; SILVA, F. S.; PORTO, A. G.. Influência das características do ar na cinética de secagem de pimenta variedade bico. Brazilian Journal of Food Technology, v.18, n.2, p.146-154, 2015.

RODRIGUES, E. A.. Avaliação dos resíduos gerados no processo produtivo de pescado na Colônia de Pescadores Z3, Pelotas - RS. Dissertação (Graduação em Engenharia Ambiental e Sanitária) - Universidade Federal de Pelotas, Pelotas, 2013.

SANTOS, J. R. M.; MARTINS, J. S.; FREIRE, M. S.; NETO, N. D. S.; SILVA, T. N.; SANTOS, J. C. O.. Caracterização físicoquímica do óleo de coco obtido artesanalmente. In: Congresso Norte - Nordeste de Química, 5. Anais. Natal: UFCG, 2013.

SILVA, E. D. P.; ALMEIDA, R. D. S.; MONTEIRO, E. A.; SILVA, H. M. G.; ROCHA, T. M.. Determinação do índice de acidez em óleo de milho para produção de biodiesel. In: Congresso Norte e Nordeste de pesquisa e inovação, 5. Anais. Maceió: IFMA, 2010.

SILVA, J. R.; RABENSCHLAG, D. R.; FEIDEN, A.; BOSCOLO, W. R.; SIGNOR, A. A.; BUENO, G. W.. Produção de Pacu em Tanques-rede no Reservatório de itaipu, Brasil: retorno econômico. Archivos de zootecnia, v.61, n.234, p.245-254, 2011.

SOBUKOLA, O. P.; OLATUNDE, S. O.. Effect of salting techniques on salt uptake and drying kinetics of African catfish (Clarias gariepinus). Food and Bioproducts Processing, v.89, n.3, p.170-177, 2011.

TOFANINI, A. J.. Controle de Qualidade de Óleos Comestíveis. Dissertação (Graduação em Química) Universidade Federal de Santa Catarina, Florianópolis, 2004. 